\title{
Optimization of urea linker of triazolopyridazine MMV665917 results in new anticryptosporidial lead with improved potency and predicted hERG safety margin
}

\author{
Edmund Oboh, ${ }^{a}$ Tanner J. Schubert, ${ }^{a}$ Jose E. Teixeira, ${ }^{b}$ Erin E. Stebbins, ${ }^{b}$ Peter Miller, ${ }^{b}$ Emily \\ Philo, ${ }^{a}$ Haresh Thakellapalli, ${ }^{a}$ Scott D. Campbell, ${ }^{c}$ David W. Griggs, ${ }^{c, d}$ Christopher D. Huston, ${ }^{b *}$ \\ and Marvin J. Meyers ${ }^{a, d *}$ \\ ${ }^{a}$ Department of Chemistry, Saint Louis University, Saint Louis, MO 63103 \\ ${ }^{b}$ Department of Medicine, University of Vermont Larner College of Medicine, Burlington, VT \\ 05401 \\ ${ }^{c}$ Department of Molecular Microbiology and Immunology, Saint Louis University School of \\ Medicine, Saint Louis, MO 63104 \\ ${ }^{d}$ Institute for Drug and Biotherapeutic Innovation, Saint Louis University, St. Louis, MO 63103
}

\section{Table of Contents}

${ }^{1} \mathrm{H}$ NMR, ${ }^{13} \mathrm{C}$ NMR, and HPLC data for compound 12a/SLU-2633 S2

Profile of 12a/SLU-2633 in the Safety47 Assay Panel S4 


\section{${ }^{1} \mathrm{H}-\mathrm{NMR}$ and ${ }^{13} \mathrm{C}$ spectra of 12a (SLU-2633)}
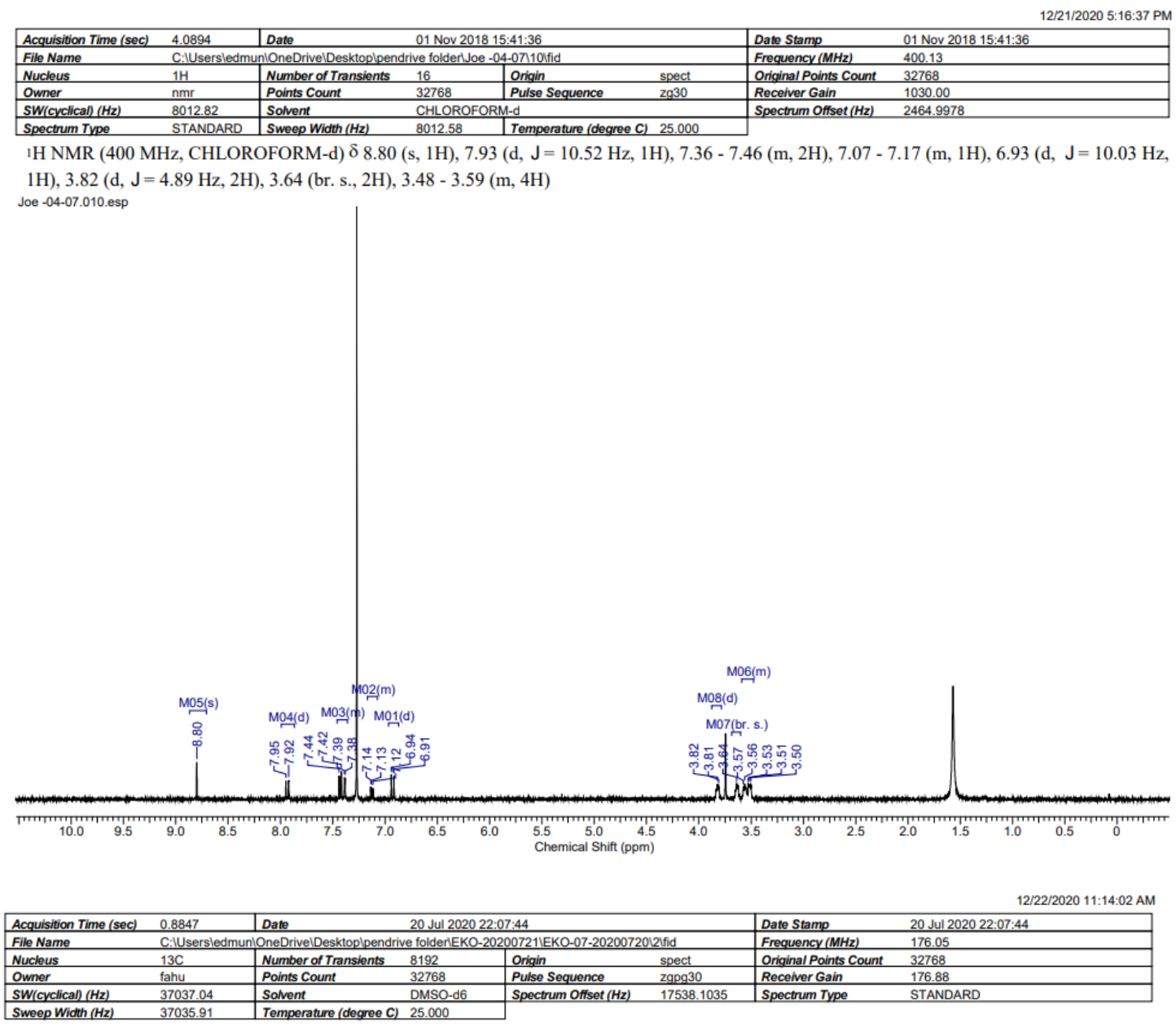

$\begin{array}{lll}\text { Sweep Width }(\mathrm{Hz}) & 37035.91 & \text { Temperature (degree C) } 25.000\end{array}$

${ }_{13 \mathrm{C}}$ NMR $\left(176 \mathrm{MHz}\right.$, DMSO-d $\left.{ }_{6}\right) \delta 168.5,155.4,141.9,138.5,137.2,131.6,130.7,130.3,130.0,129.1,124.3,115.2,45.5,45.2,44.5,40.8,38.1$ EKO-07-20200720.002.esp






\section{LC-MS Spectra of 12a (SLU-2633)}

MS Spectrum

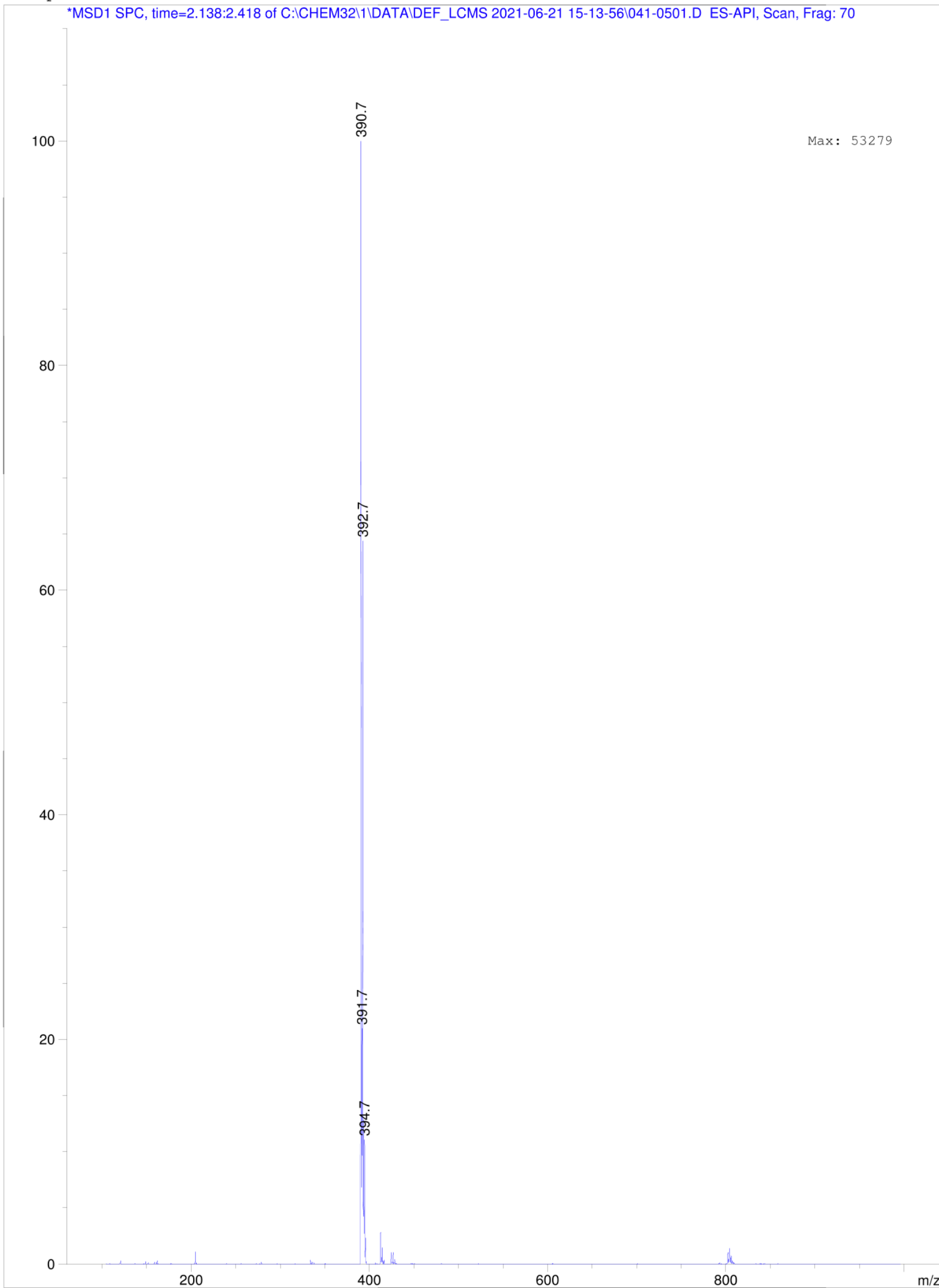


Table S1. Profile of 12a (SLU-2633) in Safety47 assay panel (Eurofins Discovery Services, St.

Charles, MO).

\begin{tabular}{|c|c|c|c|c|c|c|}
\hline Target Class & Assay Name & Assay Target & Mode & $\begin{array}{l}\text { Result } \\
\text { Type }\end{array}$ & $\begin{array}{l}\text { RC50 } \\
\text { (uM) }\end{array}$ & $\begin{array}{l}\text { Max } \\
\text { Response }\end{array}$ \\
\hline GPCR & Calcium Flux & ADORA2A & Agonist & EC50 & $>30$ & 0 \\
\hline GPCR & Calcium Flux & ADRA1A & Agonist & EC50 & $>30$ & 1.87 \\
\hline GPCR & Calcium Flux & AVPR1A & Agonist & EC50 & $>30$ & 3.15 \\
\hline GPCR & Calcium Flux & CCKAR & Agonist & EC50 & $>30$ & 3.3 \\
\hline GPCR & Calcium Flux & CHRM1 & Agonist & EC50 & $>30$ & 3.16 \\
\hline GPCR & Calcium Flux & CHRM3 & Agonist & EC50 & $>30$ & 0 \\
\hline GPCR & Calcium Flux & EDNRA & Agonist & EC50 & $>30$ & 0.54 \\
\hline GPCR & Calcium Flux & HRH1 & Agonist & EC50 & $>30$ & 1.98 \\
\hline GPCR & Calcium Flux & HTR2A & Agonist & EC50 & $>30$ & 0 \\
\hline GPCR & Calcium Flux & HTR2B & Agonist & EC50 & $>30$ & 0 \\
\hline GPCR & Calcium Flux & ADORA2A & Antagonist & IC50 & $>30$ & 6.87 \\
\hline GPCR & Calcium Flux & ADRA1A & Antagonist & IC50 & $>30$ & 16.54 \\
\hline GPCR & Calcium Flux & AVPR1A & Antagonist & IC50 & $>30$ & 0 \\
\hline GPCR & Calcium Flux & CCKAR & Antagonist & IC50 & $>30$ & 5.85 \\
\hline GPCR & Calcium Flux & CHRM1 & Antagonist & IC50 & $>30$ & 82.26 \\
\hline GPCR & Calcium Flux & CHRM3 & Antagonist & IC50 & $>30$ & 23.84 \\
\hline GPCR & Calcium Flux & EDNRA & Antagonist & IC50 & $>30$ & 0 \\
\hline GPCR & Calcium Flux & HRH1 & Antagonist & IC50 & $>30$ & 0 \\
\hline GPCR & Calcium Flux & HTR2A & Antagonist & IC50 & $>30$ & 2 \\
\hline GPCR & Calcium Flux & HTR2B & Antagonist & IC50 & $>30$ & 0 \\
\hline GPCR & cAMP & ADRA2A & Agonist & EC50 & $>30$ & 18.78 \\
\hline GPCR & cAMP & ADRB1 & Agonist & EC50 & $>30$ & 2.55 \\
\hline GPCR & cAMP & ADRB2 & Agonist & EC50 & $>30$ & 2.55 \\
\hline GPCR & cAMP & CHRM2 & Agonist & EC50 & $>30$ & 30.36 \\
\hline GPCR & cAMP & CNR1 & Agonist & EC50 & $>30$ & 0 \\
\hline GPCR & cAMP & CNR2 & Agonist & EC50 & $>30$ & 12.29 \\
\hline GPCR & cAMP & DRD1 & Agonist & EC50 & $>30$ & 4.96 \\
\hline GPCR & cAMP & DRD2S & Agonist & EC50 & $>30$ & 11.2 \\
\hline GPCR & cAMP & HRH2 & Agonist & EC50 & $>30$ & 4.01 \\
\hline GPCR & cAMP & HTR1A & Agonist & EC50 & $>30$ & 12.2 \\
\hline GPCR & cAMP & HTR1B & Agonist & EC50 & $>30$ & 9.72 \\
\hline GPCR & cAMP & OPRD1 & Agonist & EC50 & $>30$ & 5.92 \\
\hline GPCR & cAMP & OPRK1 & Agonist & EC50 & $>30$ & 8.05 \\
\hline GPCR & cAMP & OPRM1 & Agonist & EC50 & $>30$ & 7.32 \\
\hline GPCR & cAMP & ADRA2A & Antagonist & IC50 & $>30$ & 0 \\
\hline GPCR & cAMP & ADRB1 & Antagonist & IC50 & $>30$ & 26.21 \\
\hline GPCR & cAMP & ADRB2 & Antagonist & IC50 & 11.94 & 54.99 \\
\hline
\end{tabular}




\begin{tabular}{|c|c|c|c|c|c|c|}
\hline Target Class & Assay Name & Assay Target & Mode & $\begin{array}{l}\text { Result } \\
\text { Type }\end{array}$ & $\begin{array}{l}\text { RC50 } \\
\text { (uM) }\end{array}$ & $\begin{array}{l}\text { Max } \\
\text { Response }\end{array}$ \\
\hline GPCR & cAMP & CHRM2 & Antagonist & IC50 & $>30$ & 0 \\
\hline GPCR & cAMP & CNR1 & Antagonist & IC50 & $>30$ & 20.4 \\
\hline GPCR & cAMP & CNR2 & Antagonist & IC50 & $>30$ & 5.69 \\
\hline GPCR & cAMP & DRD1 & Antagonist & IC50 & $>30$ & 22.38 \\
\hline GPCR & cAMP & DRD2S & Antagonist & IC50 & $>30$ & 1.22 \\
\hline GPCR & cAMP & HRH2 & Antagonist & IC50 & $>30$ & 23.06 \\
\hline GPCR & cAMP & HTR1A & Antagonist & IC50 & $>30$ & 0.96 \\
\hline GPCR & cAMP & HTR1B & Antagonist & IC50 & $>30$ & 0.59 \\
\hline GPCR & cAMP & OPRD1 & Antagonist & IC50 & $>30$ & 0 \\
\hline GPCR & cAMP & OPRK1 & Antagonist & IC50 & $>30$ & 0.57 \\
\hline GPCR & cAMP & OPRM1 & Antagonist & IC50 & $>30$ & 0 \\
\hline Ion Channel & Ion Channel & CAV1.2 & Blocker & IC50 & $>30$ & 0 \\
\hline Ion Channel & Ion Channel & GABAA & Blocker & IC50 & $>30$ & 1.77 \\
\hline Ion Channel & Ion Channel & hERG & Blocker & IC50 & 7.707 & 79.3 \\
\hline Ion Channel & Ion Channel & HTR3A & Blocker & IC50 & $>30$ & 0 \\
\hline Ion Channel & Ion Channel & KvLQT1/minK & Blocker & IC50 & $>30$ & 0 \\
\hline Ion Channel & Ion Channel & $\mathrm{nAChR}(\mathrm{a} 4 / \mathrm{b} 2)$ & Blocker & IC50 & $>30$ & 0 \\
\hline Ion Channel & Ion Channel & NAV1.5 & Blocker & IC50 & $>30$ & 0 \\
\hline Ion Channel & Ion Channel & $\begin{array}{l}\text { NMDAR } \\
(1 \mathrm{~A} / 2 \mathrm{~B})\end{array}$ & Blocker & IC50 & $>30$ & 0.62 \\
\hline Ion Channel & Ion Channel & GABAA & Opener & EC50 & $>30$ & 0.49 \\
\hline Ion Channel & Ion Channel & HTR3A & Opener & EC50 & $>30$ & 0 \\
\hline Ion Channel & Ion Channel & KvLQT1/minK & Opener & EC50 & $>30$ & 6.7 \\
\hline Ion Channel & Ion Channel & nAChR(a4/b2) & Opener & EC50 & $>30$ & 0 \\
\hline Ion Channel & Ion Channel & $\begin{array}{l}\text { NMDAR } \\
(1 \mathrm{~A} / 2 \mathrm{~B})\end{array}$ & Opener & EC50 & $>30$ & 0 \\
\hline Kinases & Binding & INSR & Inhibitor & IC50 & $>30$ & 9.67 \\
\hline Kinases & Binding & LCK & Inhibitor & IC50 & $>30$ & 14.54 \\
\hline Kinases & Binding & ROCK1 & Inhibitor & IC50 & $>30$ & 5.33 \\
\hline Kinases & Binding & VEGFR2 & Inhibitor & IC50 & $>30$ & 13.14 \\
\hline NHR & $\begin{array}{l}\text { NHR Nuclear } \\
\text { Translocation }\end{array}$ & $\overline{\mathrm{AR}}$ & Agonist & EC50 & $>30$ & 0 \\
\hline NHR & $\begin{array}{l}\text { NHR Nuclear } \\
\text { Translocation }\end{array}$ & $\overline{A R}$ & Antagonist & IC50 & $>30$ & 0 \\
\hline NHR & $\begin{array}{l}\text { NHR Protein } \\
\text { Interaction }\end{array}$ & GR & Agonist & EC50 & $>30$ & 0 \\
\hline NHR & $\begin{array}{l}\text { NHR Protein } \\
\text { Interaction }\end{array}$ & GR & Antagonist & IC50 & $>30$ & 10.34 \\
\hline $\begin{array}{l}\text { Non-Kinase } \\
\text { Enzymes }\end{array}$ & Enzymatic & $\mathrm{AChE}$ & Inhibitor & IC50 & $>30$ & 32.67 \\
\hline $\begin{array}{l}\text { Non-Kinase } \\
\text { Enzymes }\end{array}$ & Enzymatic & COX1 & Inhibitor & IC50 & $>30$ & 0 \\
\hline $\begin{array}{l}\text { Non-Kinase } \\
\text { Enzymes }\end{array}$ & Enzymatic & COX2 & Inhibitor & IC50 & $>30$ & 0 \\
\hline
\end{tabular}




\begin{tabular}{|l|l|l|l|l|l|l|}
\hline Target Class & Assay Name & Assay Target & Mode & $\begin{array}{l}\text { Result } \\
\text { Type }\end{array}$ & $\begin{array}{l}\text { RC50 } \\
(\mathbf{u M})\end{array}$ & $\begin{array}{l}\text { Max } \\
\text { Response }\end{array}$ \\
\hline $\begin{array}{l}\text { Non-Kinase } \\
\text { Enzymes }\end{array}$ & Enzymatic & MAOA & Inhibitor & IC50 & $>30$ & 7.92 \\
\hline $\begin{array}{l}\text { Non-Kinase } \\
\text { Enzymes }\end{array}$ & Enzymatic & PDE3A & Inhibitor & IC50 & $>30$ & 2.19 \\
\hline $\begin{array}{l}\text { Non-Kinase } \\
\text { Enzymes }\end{array}$ & Enzymatic & PDE4D2 & Inhibitor & IC50 & $>30$ & 1.76 \\
\hline Transporter & Transporter & DAT & Blocker & IC50 & $>30$ & 29.87 \\
\hline Transporter & Transporter & NET & Blocker & IC50 & $>30$ & 22.64 \\
\hline Transporter & Transporter & SERT & Blocker & IC50 & $>30$ & 13.15 \\
\hline
\end{tabular}

